\title{
Acute mesenteric ischaemia: the continuing difficulty in early diagnosis
}

\author{
Steven D. Heys, Julie Brittenden and Trevor J. Crofts \\ Department of Surgery, University Medical Buildings, Foresterhill, Aberdeen AB9 2ZD, UK
}

\begin{abstract}
Summary: Five cases of acute intestinal ischaemia due to occlusion of the superior mesenteric artery, all with a delay in diagnosis, are reported here. These cases illustrate the continuing difficulties, in clinical practice, in recognizing mesenteric ischaemia before intestinal infarction has occurred, despite the clinical awareness of this condition.
\end{abstract}

\section{Introduction}

The difficulty in recognizing acute intestinal ischaemia in the early stages is well recognized because of the few clinical signs that are present and it is not until intestinal infarction has occurred that the diagnosis is made. As a result of this, the overall mortality of such patients is high (up to $80 \%$ ), and furthermore, the overall survival has changed very little over the last 35 years. ${ }^{1-4}$ However, mesenteric arterial occlusion is a condition that is amenable to surgical intervention, if it can be recognized and treated before intestinal necrosis has occurred, and therefore reduce the mortality and post-operative morbidity of those surviving patients.

We report five cases of occlusion of the superior mesenteric artery, with consequent widespread infarction of the small intestine, who presented to one surgical unit over a 12 month period. In all cases there was a significant delay in making the correct diagnosis. We examine the continuing difficulties experienced by clinicians in making an early and correct diagnosis.

\section{Case reports}

\section{Case 1}

An 82 year old man was admitted to hospital with a history of a sudden onset of colicky abdominal pain, nausea and vomiting. It was noted that the patient had a history of hypertension and a myocardial infarction 2 years previously had been noted and examination had revealed atrial fibrilla-

Correspondence: S.D. Heys, M.D., Ph.D., F.R.C.S.(Glas.) Accepted: 31 March 1992 tion. On abdominal examination there was vague central abdominal tenderness with no other signs; $\stackrel{c}{\stackrel{c}{\rho}}$ bowel sounds were normal and faecal occult blood testing was negative. Laboratory investigations revealed a white blood cell count of $15.4 \times 10^{9} \mathrm{f}$ and serum amylase was $465 \mathrm{IU} / \mathrm{l}$. A diagnosis of suspected myocardial infarction was made and the patient managed conservatively, but required nas cotic analgesics for increasing pain. In view of the persisting abdominal pain, a surgical opinion was requested. On examination (14 hours after admission to hospital), there was generalized abdominal $\stackrel{\unrhd}{\unrhd}$ tenderness with guarding and rebound, and absent $\overrightarrow{\vec{\rho}}$ bowel sounds. Plain abdominal X-rays showed several dilated small intestinal loops. A diagnosis of peritonitis, secondary to intestinal infarction, was made and laparotomy undertaken. At operation, the small intestine, extending from 2 feet distal to the duodenal-jejunal flexure to the trans- $\dot{\sigma}$ verse colon was found to be necrotic. This was resected and a primary jejuno-colic anastomosis $\delta$ performed, following which the patient made a good postoperative recovery.

\section{Case 2}

A 74 year old man was admitted to hospital, after \% being observed at home, with a 6 hour history of a $N$ sudden onset of severe epigastric pain associated $\underset{\mathrm{N}}{N}$ with profuse vomiting and a history of myocardial infarction 3 weeks previously. On examination, 0 atrial fibrillation was noted and abdominal $\underset{\Phi}{\overparen{D}}$ examination revealed distension and tenderness in $\stackrel{\mathcal{Q}}{+}$ the epigastrium, but there was no guarding or 0 rebound tenderness and bowel sounds were normal. $\bar{\partial}$

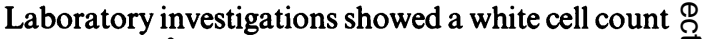
of $31.6 \times 10^{9} / 1$, serum amylase was $1,177 \mathrm{IU} / 1$ and 
plain abdominal $\mathrm{X}$-rays showed several distended loops of small bowel with air/fluid levels. A diagnosis of acute pancreatitis was made on the clinical findings in conjunction with the elevated serum amylase, and the patient managed conservatively. The patient appeared to stabilize and improve clinically. However, 6 days after admission he deteriorated suddenly, becoming hypotensive with obvious signs of generalized peritonitis. At this stage, 6 days after admission to hospital, it was decided to undertake laparotomy. At operation the jejunum, ileum and ascending colon were found to be infarcted. A resection was carried out with a primary jejuno-colic anastomosis being performed and the patient made a good postoperative recovery initially. Unfortunately, however, he died from a massive gastrointestinal haemorrhage 7 days later. A post-mortem examination revealed this to have come from a large gastric ulcer.

\section{Case 3}

A 47 year old female was admitted to hospital, after being managed at home with a 36 hour history of a sudden onset of severe colicky, central abdominal pain, associated with nausea and repeated vomiting. There was no other significant past medical history except that she was taking norethisterone for menorrhagia. On examination she was noted to be in severe pain with a sinus tachycardia (110/ minute) and a blood pressure of $110 / 65 \mathrm{mmHg}$. Abdominal examination revealed generalized tenderness and guarding with absent bowel sounds and a negative faecal occult blood test. Plain abdominal X-rays showed no abnormality, serum amylase was $372 \mathrm{IU} / 1$ and white blood cell count was $25.1 \times 10^{9} / 1$. A diagnosis of generalized peritonitis secondary to a perforated viscus was made and laparotomy undertaken. At operation the whole of the midgut extending from the second part of the duodenum to the distal transverse colon was necrotic, and was associated with a thrombus or embolus at the origin of the superior mesenteric artery. The patient subsequently died and a postmortem examination confirmed a thrombotic occlusion at the origin of the superior mesenteric artery, although no other abnormalities or predisposing factors could be found.

\section{Case 4}

A 72 year old female was admitted to hospital, after being managed at home with a 72 hour history of a sudden onset of upper abdominal colicky pain associated with nausea and vomiting. There was no significant past medical history. On examination she was centrally cyanosed, with a sinus tachycardia and systolic blood pressure of $60 \mathrm{mmHg}$. Abdominal examination revealed generalized ten- derness, rebound tenderness and guarding throughout, and faecal occult blood testing was negative. Laboratory investigations showed a white blood cell count of $17.2 \times 10^{9} / 1$, serum amylase was $451 \mathrm{IU} / 1$ and plain abdominal X-rays showed several small bowel air-fluid levels. A diagnosis of mesenteric infarction was made and, after a very short period of intensive resuscitation, she proceeded to laparotomy. At operation the whole of the small bowel was infarcted except for 2 feet of duodenum and proximal jejunum and 18 inches of terminal ileum. The cause of this was a thrombosis/embolus in the superior mesenteric artery and the necrotic intestine was resected with a primary anastomosis carried out (if there are doubts about the viability of the anastomosis, then superior mesenteric embolectomy may be worthwhile). The patient made an uneventful recovery and was discharged from hospital without any further problems.

\section{Case 5}

A 46 year old man was admitted to hospital with a 24 hour history of a sudden onset of central abdominal colicky pain, vomiting and passing blood per rectum. There was no significant past medical history except for a sudden onset of severe, central crushing chest pain, one week previously which had been diagnosed as a myocardial infarction and managed at home without hospital admission. Although the reason for the abdominal pain was unclear, he was initially managed at home until the following day when he developed a sudden onset of a cold, painful, white leg and was then referred to the Accident and Emergency Department. On examination he was hypotensive $(80 / 40$ $\mathrm{mmHg}$ ), with a sinus tachycardia of 120 /minute. Abdominal examination confirmed a generalized peritonitis with absent bowel sounds and examination of his leg revealed an arterial occlusion distal to the femoral artery. Investigations revealed a white blood cell count of $9.2 \times 10^{9} / 1$, but plain radiography of the abdomen was not carried out. He was resuscitated and proceeded to laparotomy, where the whole of the small bowel except for 12 inches of proximal jejunum was infarcted. All this small bowel was resected, after exploration and removal of an embolus from the superior mesenteric artery had failed to improve the situation. In addition, he underwent a femoral embolectomy, with the successful restoration of arterial circulation to the leg, and made a good recovery.

\section{Discussion}

The peak incidence of mesenteric infarction is in the six and seventh decades and there appears to be 
an equal male to female distribution, ${ }^{2,5}$ although some studies have found a male preponderance. ${ }^{6,7}$ Mesenteric infarction may occur not only as a result of occlusion of the superior mesenteric artery, but also because of thrombosis of the superior mesenteric vein and also 'spasm' of the intestinal circulation. There are certain causal and predisposing factors for mesenteric infarction (Table I) but in up to $50 \%$ of patients with mesenteric infarction, no obvious cause can be found. ${ }^{8.9}$

The mortality of patients who experience mesenteric infarction continues to be high, with up to $80 \%$ of such patients dying. ${ }^{1-4}$ This may be explained, in part, by the continuing difficulties the clinician has in making the correct diagnosis at an early stage, before intestinal infarction has occurred and the patient has become septicaemic with its attendant complications and sequelae. The small intestine can withstand only up to 6 hours of ischaemia before irreversible changes occur, ${ }^{10}$ and the shorter the duration of symptoms the more likely is the patient to survive after surgery. ${ }^{9}$

In the early stages of mesenteric ischaemia the patients complain of upper or central abdominal pain, which is frequently colicky and of a sudden onset associated with vomiting, and often cold sweating which is believed to be due to massive sympathetic nervous activity. ${ }^{3}$ However, abdominal examination frequently reveals minimal signs and it is not until intestinal infarction has taken place that there are then the more easily recognized signs of generalized or localized peritonitis. Often great emphasis is placed on the bowel sounds but these are present, and often hyperactive, at the onset of mesenteric ischaemia. They are only absent when intestinal infarction has occurred.

Table I Type and risk factors for mesenteric infarction

\begin{tabular}{cl}
\hline Type & Risk factors \\
\hline $\begin{array}{c}\text { Superior mesenteric } \\
\text { artery thrombosis }\end{array}$ & $\begin{array}{l}\text { Atheroma, congestive cardiac } \\
\text { failure, hypotension, oral } \\
\text { contraceptives } \\
\text { Myocardial infarction, } \\
\text { arrhythmias, ventricular } \\
\text { artery embolus } \\
\text { aneurysm, endocarditis, } \\
\text { atheroma }\end{array}$ \\
$\begin{array}{c}\text { Superior mesenteric } \\
\text { venous thrombosis }\end{array}$ & $\begin{array}{l}\text { Primary mesenteric occlusion, } \\
\text { sepsis, oral contraceptives, } \\
\text { thrombocytosis, disseminated } \\
\text { intravascular coagulation, } \\
\text { postsplenectomy } \\
\text { Digoxin or propranolol over- } \\
\text { dose, hypotension, sepsis, } \\
\text { shock, myocardial infarction } \\
\text { Unknown }\end{array}$ \\
Non-occlusive & infarction
\end{tabular}

Patients are frequently apyrexial and up to half experience loose bowel movements initially, prob- 3 ably due to increased intestinal activity. ${ }^{3}$ Faecal $\stackrel{\mathbb{Q}}{\varrho}$ occult blood testing is usually negative in the early $c$. stages of ischaemia and it is not until mucosal $\vec{\Rightarrow}$ infarction has occurred that it becomes positive. $\stackrel{5}{\stackrel{9}{+}}$ Hypotension and shock is also common, initially due to a loss of intravascular and extracellular fluid $\frac{\bar{\sigma}}{\overline{0}}$ into the intestinal lumen, ${ }^{7}$ although later septi- $\frac{\bar{\sigma}}{\vec{D}}$ caemic shock may occur because of intestinal $\stackrel{\mathbb{Q}}{\Omega}$ necrosis and the resultant translocation of intestinal bacteria form the gut lumen into the systemic $\vec{\circ}$ circulation.

All the patients in our series had complained of a $\overrightarrow{\vec{\omega}}$ sudden onset of central or upper abdominal pain $\stackrel{\rho}{\circ}$ and all of them (except one in whom there was no 8 known cause), had well-recognized risk factors for 3 . mesenteric infarction - atrial fibrillation, recent 8 myocardial infarction and hormone replacement $\infty$ therapy with progestogens alone. However, despite their clinical histories and the presence of clearly $\underset{\infty}{\stackrel{p}{0}}$ identifiable risk factors, there was a mean time delay in the diagnosis of mesenteric infarction, by surgeons, physicians and general practitioners, of $c$ 50 hours (range 16-104 hours) in these patients.

Although the importance of a thorough clinical history and examination cannot be understated, it would be of considerable help if there were laborig tory investigations that which could be of help. The $\omega$ small intestine is a rich source of phosphate and experimental animal studies have shown that in the early stages of intestinal ischaemia and before necrosis has supervened, the serum phosphate is significantly elevated. This has been evaluated in patients with mesenteric infarction, with $80 \%$ having elevations in serum phosphate, although bowel infarction had occurred. ${ }^{11}$ In addition, these authors found that there was an associated metabolic acidosis and leucocytosis and they believed that this triad strongly supported a diagnosis of intestinal ischaemia. ${ }^{10}$ It is important to note that serum amylase may also be elevated in approximately half of patients with intestinal infarction, and in $6 \%$ of them can be elevated to such levels that a misdiagnosis of acute pancreatitis might be made. ${ }^{9}$ Indeed, patient 2 in our series had a greatly elevated amylase and a diagnosis of pancreatitis was made. This had resulted in laparotomy being delayed until 6 days after the patient had been o admitted to hospital. Therefore, if patients in N whom a diagnosis of acute pancreatitis has been $\underset{\mathrm{N}}{\mathrm{N}}$ made fail to settle adequately, then mesenteric infarction must be considered. Plain radiographs of the abdomen may not be helpful; some patients may have air fluid levels in small and/or large $\stackrel{?}{?}$ intestine in the early stages, but more than one third 7 of the patients have no abnormality on first presentation. ${ }^{3}$ Angiography may have a role to play in specialized centres where it is readily available, 
although there are drawbacks - the risk of the procedure, negative investigations and finding an already occluded artery which had been asymptomatic. More recently, experimental studies have been investigating the use of magnetic resonance imaging as a non-invasive method of diagnosing intestinal ischaemia ${ }^{12}$ but this requires thorough evaluation before applied to man in the clinical situation.

Clearly, in retrospect, the diagnosis of mesenteric infarction should have been made readily in four of the five cases, but the key to early diagnosis must lie in a high index of suspicion of this condition, especially when the severity of the abdominal pain exceeds the physical signs and

\section{References}

1. Shaw, R.S. \& Rutledge, R.H. Superior mesenteric artery embolectomy in the treatment of massive mesenteric infarction. $N$ Engl J Med 1957, 257: 595-598.

2. Ottinger, L.W. The surgical management of acute occlusion of the superior mesenteric artery. Ann Surg 1978, 188: 721-731.

3. Krausz, M.M. \& Manny, J. Acute superior mesenteric arterial occlusion: a plea for early diagnosis. Surgery 1978 , 83: $482-485$.

4. Andersson, R., Parsson, H., Isaksson, B. \& Norgren, L. Acute intestinal ischaemia. A 14 year retrospective investigation. Acta Chir Scand 1984, 150: 217-221.

5. Sachs, S.M., Morton, J.H. \& Schwartz, S.I. Acute mesenteric ischaemia. Surgery 1982, 92: 646-653.

6. Rius, X., Escalante, J.F., Llaurado, J.M., Jover, J. \& Lacalle, J. Mesenteric infarction. World J Surg 1979, 3: 489-496.

7. Sitges-Serra, A., Mas, X., Roqueta, F., Figueras, J. \& Sanz, F. Mesenteric infarction: an analysis of 83 patients with prognostic studies in $\mathbf{4 4}$ cases undergoing a massive small-bowel resection. Br J Surg 1988, 75: 544-548. when there are known risk factors present. At the present time there are no reliable laboratory indicators of intestinal ischaemia and a heightened awareness of the clinical problem in conjunction with early access to angiography represent two significant factors in improving management of this surgical emergency.

\section{Acknowledgements}

We would like to thank the various consultants concerned for permission to report their patients and Professor O. Eremin for reading the manuscript.

8. Ottinger, L.W. \& Austen, W.G. A study of 136 patients with mesenteric infarction. Surg Gynecol Obstet 1967, 124: 251261

9. Wilson, C., Gupta, R., Gilmour, D.G. \& Imrie, C.W. Acute superior mesenteric ischaemia. Br J Surg 1987, 74: 279-281.

10. Jones, P.F. Emergency Abdominal Surgery. Blackwell Scientific Publications, Oxford, 1987, pp. 481-487.

11. Jamieson, W.G., Marchuk, S., Rowsom, J. \& Durand, D. The early diagnosis of massive intestinal ischaemia. $\mathrm{Br} J$ Surg 1982, 69 (Suppl): S52-S53.

12. Temes, R.T., Kauten, R.J. \& Schwartz, M.Z. Nuclear magnetic resonance as a noninvasive method of diagnosing intestinal ischaemia: technique and preliminary results. $J$ Pediatr Surg 1991, 26: 775-779. 